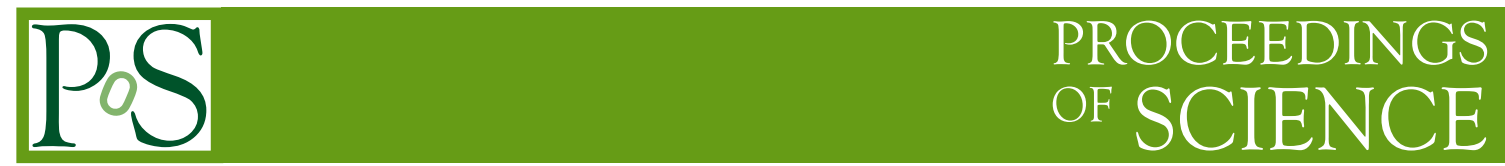

\title{
CKM session summary
}

\section{Marta Calvi*}

Univerity Milano Bicocca and INFN

E-mail: marta.calvi@mib.infn.it

A short summary of the CKM session is provided here, giving an overview of the status of current results and of future perspectives.

The Xth Nicola Cabibbo International Conference on Heavy Quarks and Leptons, October 11-15, 2010

Frascati (Rome) Italy

${ }^{*}$ Speaker. 


\section{Introduction}

The elements of the Cabibbo-Kobayashi-Maskawa (CKM) matrix, which describe the couplings of the charged weak current to quarks, are under study since years. As it was shown at this Conference, theoretical calculations and measurements from many experiments, provided with continuously improving precision, have defined a complete picture which is in general good agreement with Standard Model (SM) predictions. However, from a closer look, there are also areas where some tension exists between different results. Additional input is required from updates on current measurements and from new experiments to resolve present ambiguities and unravel possible effects of New Physics (NP). A brief summary of the current status is given here, as an introduction to the full collection of contributions included in this section.

\section{CKM parameters}

Stringent tests of unitarity are possible on the first row of the CKM matrix, which represent a tight constraint for any NP model. Currently the most precise measurement of $\left|V_{u s}\right|$ comes from $K_{l 3}$ decays, as was shown by C.Bloise [1]. An alternative way to determine this CKM element come from tau decays (I. Nugent [2]). The strange and non-strange inclusive and exclusive tau hadronic widths allow to extract $\left|V_{u s}\right|$ with several techniques, and using different theoretical inputs. Babar and Belle have already measured nine of the main tau strange branching fractions. The Finite Energy Sum Rules approach is the one with the smallest theoretical error (0.23-0.47\%). It currently gives $\left|V_{u s}\right|=0.2188 \pm 0.0023$ and has the potential to become, with updated experimental inputs, the most precise measurement of $\left|V_{u s}\right|$.

For the determination of $\left|V_{u b}\right|$ the measurement of the inclusive charmless semi-leptonic decay is used and the challenge is the suppression of the background from $B \rightarrow X_{c} \ell v$ decays, about fifty times higher. As a consequence, most analysis have been performed in the past years in the restricted phase space region accessible to signal decays. A theoretical parametrization (Shape Functions) is needed to describe the unmeasured region, and it is the main source of uncertainty on the $\left|V_{u b}\right|$ exclusive measurement. To overcome this problem, two recent inclusive measurements from Babar and Belle, reported by N. Gagliardi [3], have extended the analysis to about $90 \%$ of the phase space. The corresponding finally uncertainty on $\left|V_{u b}\right|$ is about $8 \%$ for both experiments. I. Nakamura [4] presented the new preliminary untagged analysis from Belle of the exclusive $\left|V_{u b}\right|$ from the partial branching fraction $B^{0} \rightarrow \pi^{-} \ell^{+} v$. The agreement between inclusive and exclusive measurements remains unsatisfactory, as since several years.

The semi-leptonic $B \rightarrow X_{c} \ell \nu$ inclusive decays, together with the radiative $B \rightarrow X_{s} \gamma$ decay, allow the extraction of the CKM element $\left|V_{c b}\right|$ together with the determination of other fundamental parameters like the $b$ quark mass $m_{b}$ and the parameters of the HQE $\left(\mu_{\pi}^{2}, \mu_{G}^{2}, \rho_{L S}^{3}\right.$ and $\left.\rho_{D}^{3}\right)$ (K. Knoepfel [5]). A precision of $2 \%$ on $\left|V_{c b}\right|$ has now been achieved. Also in the case of $\left|V_{c b}\right|$ there remain a small discrepancy between the inclusive measurement and the value extracted from measurements of the B exclusive decays ( [4]).

A demonstration of the excellent overall consistency of all available CKM measurements has been shown by C.Tarantino [6] with the Unitarity Triangle analysis. The fits performed in the SM framework, where no NP effects are allowed and all available constraints are included, over- 
constraint the parameters of the UT. The apex position is accurately determined as: $\bar{\rho}=0.132 \pm$ $0.020, \bar{\eta}=0.358 \pm 0.012$. It is thus established that the CKM matrix is the dominant source of flavour mixing and $\mathrm{CP}$ violation and NP effects can provide only small correction to this picture.

The fit is repeated using only tree-level constraints, namely $\left|V_{u b}\right| /\left|V_{c b}\right|$ from B semi-leptonic decays and the measurements of the angle $\gamma$ from non-leptonic tree-level decays $B \rightarrow D^{(*)} K$, allowing for NP effects to affect in the most general way all loop process. Results are still compatible with those of the SM fit, but with much larger uncertainties. They will be strongly reduced by new measurements on $\gamma$ foreseen in the future, for example at the LHCb experiment.

The precision of UT fits has increased also thanks to the improvements in Lattice QCD calculation, as was discussed by V.Lubicz [7]. In the last four years there has been a gain of a factor two or more in the precision on the calculation of some parameters like $\left.f_{+}(0), B_{K}, f_{B} \sqrt{(} B_{B}\right), f_{B s}$ $\left.\sqrt{(} B_{B s}\right)$, which has been crucial for many tests. This was due to the increased computational power of TeraFlops machines, required to perform un-quenched simulations, but also from improvements in the used algorithms. In many cases another step is still needed to reach the current experimental precision and the effort is ongoing.

Within the UT analysis in the SM framework two results demand further investigation. One is related to the current 2.6 sigma deviation between the prediction of $\sin 2 \beta=0.771 \pm 0.036$ and the world average measurement $\sin 2 \beta=0.654 \pm 0.026$.

The second is the deviation between the predicted branching fraction $B R(B \rightarrow \tau \nu)=0.805 \pm$ $0.071 \times 10^{-4}$ and the experimental measurements $B R(B \rightarrow \tau v)=1.64 \pm 0.34 \times 10^{-4}$. This is the overall average between measurements from Babar and Belle including the analysis using both hadronic and leptonic tags, which was presented by D.Lindermann [8]. The SM rate could be enhanced or suppressed by the presence of a virtual charged Higgs boson replacing the $W^{+}$boson in the annihilation diagram. Hence the measurement of this branching ratio can be used to put limits on NP, as an example on the mass of the charged Higgs in type-II two Higgs doublet model $(2 \mathrm{HDM})$ as a function of $\tan \beta$. Similar constraints can be extracted from the semi-leptonic decay $B \rightarrow D^{(*)} \tau \nu$ which is also sensitive to a charged Higgs coupling at tree level, with the advantage of a predicted branching fraction which is in the SM four orders of magnitude larger than for the leptonic decay. Both Babar and Belle have recently updated their analysis of these decay modes, including $B^{+} \rightarrow D^{(*) 0} \tau^{+} v$ and $B^{0} \rightarrow D^{(*)^{-}} \tau^{+} v$ channels.

The leptonic decays $B^{+} \rightarrow \ell v$, with $\ell=e, \mu$ are less accessible than $\tau$ decays, due to the branching fraction's proportionality to the square of the lepton mass, but they can provide clean measurements and good tests for the presence of effects of NP. The recent upper limit from Babar $B R\left(B^{+} \rightarrow \mu^{+} v\right)<1.0 \times 10^{-6}$ at $90 \% \mathrm{CL}$ approaches the SM expectation which is $\sim 5 \times 10^{-7}$, while the current best limit for the decay into electron, from Belle: $B R\left(B^{+} \rightarrow e^{+} v\right)<0.98 \times 10^{-6}$ at $90 \% \mathrm{CL}$ is still orders of magnitude above the SM expectation of $10^{-11}$. A step forward in the measurement of these rare B decays will come in next years from Super B factories. Perspective for the precision foreseen with up to $75 \mathrm{ab}^{-1}$ of data were discussed in the contribution of A. Perez [9].

\section{References}

[1] C. Bloise in these proceedings.

[2] I. Nugent in these proceedings. 
[3] N. Gagliardi in these proceedings.

[4] I. Nakamura in these proceedings.

[5] K. Knoepfel in these proceedings.

[6] C. Tarantino in these proceedings.

[7] V. Lubicz in these proceedings.

[8] D. Lindermann in these proceedings.

[9] L. A. Perez in these proceedings. 